\title{
Geometric Phases, Reduction and Lie-Poisson Structure for the Resonant Three-wave Interaction*
}

\author{
Mark S. Alber ${ }^{\dagger}$ \\ Department of Mathematics \\ University of Notre Dame \\ Notre Dame, IN 46556 \\ email: Mark.S.Alber.1@nd.edu \\ Gregory G. Luther $\ddagger$ \\ Engineering Sciences and Applied Mathematics Department \\ McCormick School of Engineering and Applied Science \\ Northwestern University \\ 2145 Sheridan Road \\ Evanston, Il 60208-3125. \\ email: ggluther@nwu.edu \\ Jerrold E. Marsden ${ }^{\S}$ \\ Control and Dynamical Systems 107-81 \\ Caltech, \\ Pasadena, CA 91125 \\ email: marsden@cds.caltech.edu \\ Jonathan M. Robbins $₫$ \\ The Basic Research Institute in the Mathematical Sciences (BRIMS) \\ Hewlett-Packard Laboratories \\ Filton Road, Stoke Gifford \\ Bristol BS12 6QZ, UK \\ and \\ School of Mathematics \\ University Walk, Bristol BS8 1TW UK \\ email: j.robbins@bristol.ac.uk \\ Spring, 1995; this version, January 30, 1998
}




\begin{abstract}
Hamiltonian Lie-Poisson structures of the three-wave equations associated with the Lie algebras $\mathfrak{s u}(3)$ and $\mathfrak{s u}(2,1)$ are derived and shown to be compatible. Poisson reduction is performed using the method of invariants and geometric phases associated with the reconstruction are calculated. These results can be applied to applications of nonlinear-waves in, for instance, nonlinear optics. Some of the general structures presented in the latter part of this paper are implicit in the literature; our purpose is to put the three-wave interaction in the modern setting of geometric mechanics and to explore some new things, such as explicit geometric phase formulas, as well as some old things, such as integrability, in this context.
\end{abstract}

\title{
Contents
}

1 Introduction 3

2 The Canonical Hamiltonian Structure. $\quad 5$

$\begin{array}{lll}3 & \text { Poisson Reduction } & 7\end{array}$

4 Three-wave phase formulas $\quad 9$

4.1 The general case. . . . . . . . . . . . . . . . . 10

4.2 The case of second harmonic generation. . . . . . . . . . . . . 14

5 The Lie-Poisson Structure $\quad 17$

6 Connections between the two Hamiltonian structures 19

7 Discussion $\quad 23$

*Keywords: Geometric phase, integrable systems, Lax equation, Lie-Poisson, nonlinear waves, reduction, three wave interaction; PACS Numbers: 4.30.Nk, 3.40.Kf, 42.65.-k, 3.40.Gc, 52.35.Mw

${ }^{\dagger}$ Research partially supported by NSF grants DMS 9626672 and 9508711.

${ }^{\ddagger}$ GGL gratefully acknowledges support from BRIMS, Hewlett-Packard Labs and from NSF DMS under grants 9626672 and 9508711.

${ }^{\S}$ Research partially supported by the Department of Energy under Contract DE-FG0395ER25251.

『Research partially supported by NSF grant DMS 9508711, NATO grant CRG 950897 and by the Department of Mathematics and the Center for Applied Mathematics, University of Notre Dame 


\section{Introduction}

Resonant wave interactions permit the exchange of wave action or energy among nonlinear modes in a variety of physical systems. For instance, the three-wave interaction occurs when the wave numbers and frequencies of three nonlinear waves satisfy either the matching conditions $\mathbf{k}_{1}=\mathbf{k}_{2}-\mathbf{k}_{3}$, and $\omega_{1}=\omega_{2}-\omega_{3}$ for a decay process or the matching conditions $\mathbf{k}_{1}=-\mathbf{k}_{2}-\mathbf{k}_{3}$, and $\omega_{1}=-\omega_{2}-\omega_{3}$ for an explosive process. The three-wave equations describe the resonant quadratic nonlinear interaction of three waves and are obtained as amplitude equations in an asymptotic reduction of primitive equations in optics, fluid dynamics and plasma physics.

The purely quadratic three-wave system of ordinary differential equations is

$$
\begin{aligned}
& \frac{d q_{1}}{d t}=i s_{1} \gamma_{1} q_{2} \bar{q}_{3} \\
& \frac{d q_{2}}{d t}=i s_{2} \gamma_{2} q_{1} q_{3} \\
& \frac{d q_{3}}{d t}=i s_{3} \gamma_{3} \bar{q}_{1} q_{2}
\end{aligned}
$$

Here, the $\gamma_{i}$ are nonzero real numbers such that $\gamma_{1}+\gamma_{2}+\gamma_{3}=0$. Each $q_{i} \in \mathbb{C}$, so these are systems of ordinary differential equations on $\mathbb{C}^{3}$. For a fixed choice of the $\gamma_{j}$, the choice of signs, determined by $\left(s_{1}, s_{2}, s_{3}\right)$ distinguishes between three decay interactions which have bounded solutions in time and an explosive interaction which has solutions that blow up in finite time. Choosing $\gamma_{1}, \gamma_{3}>0$ and $\gamma_{2}<0$, the decay systems are obtained by choosing $\left(s_{1}, s_{2}, s_{3}\right)$ as $(1,-1,1),(1,-1,-1)$ or $(-1,-1,1)$. The explosive system is obtained by choosing $(-1,-1,-1)$.

These systems are represented below as Lie-Poisson equations for the groups $\mathrm{SU}(3)$ and $\mathrm{SU}(2,1)$.

Decay Interaction. One of these is obtained by choosing

$$
\left(s_{1}, s_{2}, s_{3}\right)=(1,-1,1) \text { and }\left(\gamma_{1}, \gamma_{2}, \gamma_{3}\right)=(1,-2,1) .
$$

After changing variables to $Q_{1}=\sqrt{2} q_{1}, Q_{2}=q_{2}$ and $Q_{3}=\sqrt{2} q_{3},(1.1)$ takes the standard form

$$
\begin{aligned}
& \frac{d Q_{1}}{d t}=i Q_{2} \bar{Q}_{3} \\
& \frac{d Q_{2}}{d t}=i Q_{1} Q_{3} \\
& \frac{d Q_{3}}{d t}=i Q_{1} \bar{Q}_{2}
\end{aligned}
$$

and models the dynamics of three resonantly coupled positive-energy waves. All solutions in $\mathbb{C}^{3}$ remain uniformly bounded. 
Explosive Interaction. This is obtained by choosing

$$
\left(s_{1}, s_{2}, s_{3}\right)=(-1,-1,-1) \quad \text { and } \quad\left(\gamma_{1}, \gamma_{2}, \gamma_{3}\right)=(1,-2,1)
$$

After changing variables to $Q_{1}=\sqrt{2} \bar{q}_{1}, Q_{2}=q_{2}$ and $Q_{3}=\sqrt{2} \bar{q}_{3}$, this system takes the standard form

$$
\begin{aligned}
& \frac{d Q_{1}}{d t}=i \bar{Q}_{2} \bar{Q}_{3}, \\
& \frac{d Q_{2}}{d t}=i \bar{Q}_{1} \bar{Q}_{3}, \\
& \frac{d Q_{3}}{d t}=i \bar{Q}_{1} \bar{Q}_{2},
\end{aligned}
$$

and models the dynamics of three resonantly coupled waves with indefinite sign for each conserved quantity. Solutions in $\mathbb{C}^{3}$ can blow up in finite time. (see Zakharov and Manakov [1] or Ablowitz and Segur [2]).

Basic wave interactions of this kind are fundamental in the understanding and analysis of a variety of phenomena including patterns, symmetry induced instabilities, the Benjamin-Feir instability and many others. The three-wave equations are closely related to the equations governing coupled harmonic oscillators, tops, the rigid body and even to the traveling wave solutions of the Maxwell-Bloch equations [3] governing the interaction of light with a two-level atom. This is understood by realizing that the three-wave equations are the complex equations for a resonant three degree of freedom Hamiltonian system. They contain the Euler equations (see for instance Guckenheimer and Mahalov [4]) associated with $\mathrm{SO}(3)$ as a real subspace of SU(3). The Maxwell-Bloch equations are also contained in the three-wave equations (see David and Holm [5] for details). Some general references to literature on the integrable three-wave equations is found in Whitham [6] Ablowitz and Haberman [7], Kaup [8, 9, 10], Zakharov and Manakov [11], Ablowitz and Segur [2], Newell [12], and Ablowitz and Clarkson [13].

The integrable Hamiltonian structure of the three-wave equations is of course well known; we explore it from a somewhat novel point of view in what follows. As we will show, these equations possess a Lie-Poisson structure in addition to the canonical Hamiltonian structure. One of the three-wave decay systems is LiePoisson for the Lie algebra $\mathfrak{s u}(3)$. Two of the decay systems and the explosive threewave system are Lie-Poisson for $\mathfrak{s u}(2,1)$. Using the method of translation of the argument, two compatible Hamiltonian structures are obtained. One is the canonical Hamiltonian structure embedded in $\mathfrak{s u}(3)$ or $\mathfrak{s u}(2,1)$; it has a cubic Hamiltonian. The other is non-canonical having a standard left invariant Lie-Poisson bracket; it has a quadratic Hamiltonian. These two Poisson brackets lead to a recursion relation that is expressed in terms of Lie brackets. This recursion relation is the same one that is found using the Lax pair approach.

Solutions for the integrable three-wave equations and other similar systems are well known. In our approach below, they are reduced and integrated using a pair of $S^{1}$ actions, the canonical Hamiltonian structure and the technique of invariants. In solving the reconstruction problem, phase formulas analogous to those obtained for 
the rigid body [14] are obtained. These formulas give the value of the phase shifts that accompany the periodic exchange of wave action in resonant wave systems (see for instance $[15,16,17,18,19])$.

Though the main development is restricted to the three-wave system, we remark that as in the case of the $n$-component Euler equations [20], all of the basic results described below generalize to $n$-wave systems (see Kummer [21] for a treatment of reduction for the $n$-degree of freedom Hamiltonian with resonances). The structure of the $n$-wave interaction is related to the family of Lie algebras in $\mathfrak{s u}(n)$ or $\mathfrak{s u}(p, q)$.

The general picture developed here is useful for many other purposes, including polarization control (building on work of Holm, David and Tratnik [22]) and perturbations of Hamiltonian normal forms (see work of Knobloch, Mahalov and Marsden [23], Kirk, Marsden and Silber [24], and Haller and Wiggins [25]). They have also been used to analyze quasi-phase-matched second harmonic generation [26] in nonlinear optics where the transfer of energy or wave action among the waves is controlled by modulating the quadratic nonlinearity.

\section{The Canonical Hamiltonian Structure.}

A $\gamma_{i}$-weighted canonical Poisson bracket on $\mathbb{C}^{3}$ is used. This bracket has the real and imaginary parts of each complex dynamical variable $q_{i}$ as conjugate variables. The Hamiltonian for the three-wave equations is cubic in this setting.

The Canonical Symplectic and Poisson Structure. Writing $q_{k}=x_{k}+i y_{k}$ and treating $x_{k}$ and $y_{k}$ as conjugate variables, the (scaled) canonical Poisson bracket is given by

$$
\{F, G\}=\sum_{k=1}^{3} s_{k} \gamma_{k}\left(\frac{\partial F}{\partial x_{k}} \frac{\partial G}{\partial y_{k}}-\frac{\partial G}{\partial x_{k}} \frac{\partial F}{\partial y_{k}}\right) .
$$

In standard matrix notation this is

$$
\{F, K\}=(\nabla F) \mathbb{J}(\nabla K),
$$

where the gradients are standard gradients in $\mathbb{R}^{6}$ (with the variables ordered as $\left.\left(x_{1}, x_{2}, x_{3}, y_{1}, y_{2}, y_{3}\right)\right)$ and where

$$
\mathbb{J}=\left(\begin{array}{cc}
0 & \Gamma \\
-\Gamma & 0
\end{array}\right)
$$

and $\Gamma$ is the $3 \times 3$ matrix with $s_{k} \gamma_{k}$ on the diagonal and zeros elsewhere.

This bracket may be written in complex notation as

$$
\{F, G\}=-2 i \sum_{k=1}^{3} s_{k} \gamma_{k}\left(\frac{\partial F}{\partial q^{k}} \frac{\partial G}{\partial \bar{q}_{k}}-\frac{\partial G}{\partial q^{k}} \frac{\partial F}{\partial \bar{q}_{k}}\right) .
$$

The corresponding symplectic structure is written as follows:

$$
\omega\left(\left(z_{1}, z_{2}, z_{3}\right),\left(w_{1}, w_{2}, w_{3}\right)\right)=-\sum_{k=1}^{3} \frac{1}{s_{k} \gamma_{k}} \operatorname{Im}\left(z_{k} \bar{w}_{k}\right) .
$$


The Hamiltonian. The Hamiltonian for the three-wave interaction is

$$
H_{3}=-\frac{1}{2}\left(\bar{q}_{1} q_{2} \bar{q}_{3}+q_{1} \bar{q}_{2} q_{3}\right) \text {. }
$$

Hamilton's equations are

$$
\frac{d q_{k}}{d t}=\left\{q_{k}, H\right\}
$$

and it is straightforward to check that Hamilton's equations are given in complex notation by

$$
\frac{d q_{k}}{d t}=-2 i s_{k} \gamma_{k} \frac{\partial H}{\partial \bar{q}_{k}} .
$$

One checks that Hamilton's equations with $H=H_{3}$ coincide with (1.1).

Therefore, the following standard result holds.

Proposition 2.1 With the preceding Hamiltonian $\mathrm{H}_{3}$ and the symplectic or equivalently the Poisson structure given above, Hamilton's equations are given by the three-wave equations (1.1).

Integrals of Motion. In addition to $H_{3}$ itself, one identifies the following constants of motion,

$$
\begin{aligned}
& K_{1}=\frac{1}{2}\left(\frac{\left|q_{1}\right|^{2}}{s_{1} \gamma_{1}}+\frac{\left|q_{2}\right|^{2}}{s_{2} \gamma_{2}}\right), \\
& K_{2}=\frac{1}{2}\left(\frac{\left|q_{2}\right|^{2}}{s_{2} \gamma_{2}}+\frac{\left|q_{3}\right|^{2}}{s_{3} \gamma_{3}}\right), \\
& K_{3}=\frac{1}{2}\left(\frac{\left|q_{1}\right|^{2}}{s_{1} \gamma_{1}}-\frac{\left|q_{3}\right|^{2}}{s_{3} \gamma_{3}}\right) .
\end{aligned}
$$

These are often referred to as the Manley-Rowe relations. The Hamiltonian with any two of the $K_{j}$ are checked to be a complete and independent set of conserved quantities in involution (the $K_{j}$ clearly give only two independent invariants since $K_{1}-K_{2}=K_{3}$ ). In the sense of Liouville-Arnold this system is therefore integrable.

To integrate the three-wave equations one typically makes use of the Hamiltonian, $H_{3}$, plus two of the integrals, $K_{j}$, to reduce the system to quadratures. This procedure is usually carried out using the transformation $q_{j}=\sqrt{\rho_{j}} \exp i \phi_{j}$. Below a different approach is described. It appears to be less cumbersome, and it provides considerable geometric insight.

First observe that the following proposition holds.

Proposition 2.2 The vector function $\left(K_{1}, K_{2}, K_{3}\right)$ is the momentum map for the following symplectic action of $T^{3}=S^{1} \times S^{1} \times S^{1}$ :

$$
\begin{aligned}
& \left(q_{1}, q_{2}, q_{3}\right) \mapsto\left(q_{1} \exp (-i \gamma), q_{2} \exp (-i \gamma), q_{3}\right), \\
& \left(q_{1}, q_{2}, q_{3}\right) \mapsto\left(q_{1}, q_{2} \exp (-i \gamma), q_{3} \exp (-i \gamma)\right), \\
& \left(q_{1}, q_{2}, q_{3}\right) \mapsto\left(q_{1} \exp (-i \gamma), q_{2}, q_{3} \exp (i \gamma)\right) .
\end{aligned}
$$


Any combination of two of these actions is generated by the third reflecting the fact that the $K_{j}$ are linearly dependent. Another way of saying this is that the group action by $T^{3}$ is really captured by the action of $T^{2}$.

\section{Poisson Reduction}

In this section symplectic and Poisson reduction are performed on the three-wave Hamiltonian system using the $S^{1}$ symmetries associated with the momentum maps $K_{k}$. In terms of Poisson reduction, the process is to replace $\mathbb{C}^{3} \rightarrow \mathbb{C}^{3} / T^{2}$. The symplectic leaves in this reduction are obtained using the method of invariants.

Invariant coordinates for three-wave reduction. Invariants for the $T^{2}$ action are:

$$
\begin{aligned}
X+i Y & =q_{1} \bar{q}_{2} q_{3}, \\
Z_{1} & =\left|q_{1}\right|^{2}-\left|q_{2}\right|^{2}, \\
Z_{2} & =\left|q_{2}\right|^{2}-\left|q_{3}\right|^{2} .
\end{aligned}
$$

These quantities provide coordinates for the four-dimensional orbit space $\mathbb{C}^{3} / T^{2}$. The coordinates, $X, Y, Z_{1}$ and $Z_{2}$ are Hopf-like variables (see, e.g., [27]) and they generalize the well known Stokes parameters (see, e.g., [28]) to resonant interactions for systems with more than two complex components.

Reduced three-wave surfaces. The following identity holds for these invariants and the conserved quantities:

$$
X^{2}+Y^{2}=\kappa_{4}\left(2 s_{2} \gamma_{2} K_{1}+Z_{1}\right)\left(2 s_{3} \gamma_{3} K_{2}+Z_{2}\right)\left(2 s_{2} \gamma_{2} K_{2}-Z_{2}\right)
$$

where $\kappa_{4}=\left(s_{1} \gamma_{1} s_{2} \gamma_{2} s_{3} \gamma_{3}\right) /\left(s_{1} \gamma_{1}+s_{2} \gamma_{2}\right)\left(s_{2} \gamma_{2}+s_{3} \gamma_{3}\right)^{2}$. Trajectories in these reduced coordinates lie on the set defined by this relation. Using the conservation laws $K_{k}$ and the definitions of $Z_{1}$ and $Z_{2}$, a second relation between $K_{1}, K_{2}$ and $Z_{1}, Z_{2}$ is identified and either of the coordinates $Z_{j}$ is removed reducing the number of real dimensions to three. In $\mathbb{R}^{3}$ the reduced trajectories lie on the invariant set:

$$
X^{2}+Y^{2}=\kappa_{3}\left(\delta-Z_{2}\right)\left(2 s_{3} \gamma_{3} K_{2}+Z_{2}\right)\left(2 s_{2} \gamma_{2} K_{2}-Z_{2}\right),
$$

where $\kappa_{3}=\left(s_{1} \gamma_{1} s_{2} \gamma_{2} s_{3} \gamma_{3}\right) /\left(s_{2} \gamma_{2}+s_{3} \gamma_{3}\right)^{3}$ and $\delta=2 s_{2} \gamma_{2} K_{1}+2 s_{3} \gamma_{3}\left(K_{1}-K_{2}\right)$. This relation defines a two dimensional (perhaps singular) surface in $\left(X, Y, Z_{2}\right)$ space, with $Z_{1}$ determined by the values of these invariants and the conserved quantities (so it may also be thought of as a surface in $\left(X, Y, Z_{1}, Z_{2}\right)$ as well). The relations between the invariants and the conserved quantities may imply inequalities for, say, $Z_{2}$; these may imply that the corresponding surface is compact. A sample of one of these surfaces is plotted in Fig. 3.1. These surfaces will be called the three-wave surfaces below. 


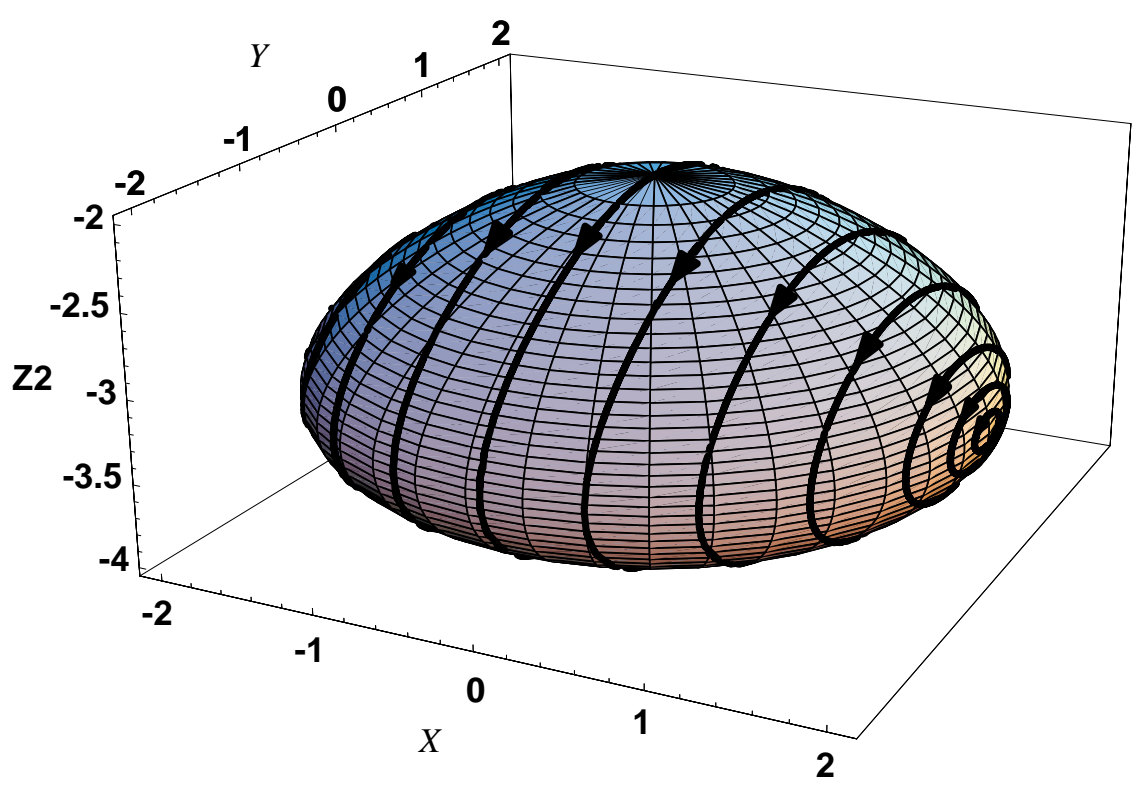

Figure 3.1: A three-wave surface is drawn in $\left(X, Y, Z_{2}\right)$ coordinates for the decay interaction. Trajectories are also drawn showing the phase space of the reduced three-wave equations on the three-wave surface when $\left(s_{1}, s_{2}, s_{3}\right)=(1,1,1)$, $\left(\gamma_{1}, \gamma_{2}, \gamma_{3}\right)=(1,1,-2)$, and $\left(K_{1}, K_{2}\right)=(1,-1 / 2)$.

Reduced three-wave equations. Any trajectory of the original equations defines a curve on each three-wave surface, in which the $K_{j}$ are set to constants. These three-wave surfaces are the symplectic leaves in the four-dimensional Poisson space having coordinates $\left(X, Y, Z_{1}, Z_{2}\right)$.

The original equations define a dynamical system in the Poisson reduced space and on the symplectic leaves as well. Using these new coordinates the Poisson bracket and the Hamiltonian are reduced directly. The reduced Hamiltonian is

$$
H_{r}=-X .
$$

With the reduced Poisson brackets in $\left(X, Y, Z_{2}\right), H_{r}$ produces the reduced equations of motion

$$
\begin{aligned}
\frac{d X}{d t} & =0, \\
\frac{d Y}{d t} & =\frac{\partial \phi}{\partial Z_{2}}, \\
\frac{d Z_{2}}{d t} & =-2\left(s_{2} \gamma_{2}+s_{3} \gamma_{3}\right) Y,
\end{aligned}
$$

where the dynamical invariant $\phi$ is defined by

$$
\begin{aligned}
\phi= & \left(s_{2} \gamma_{2}+s_{3} \gamma_{3}\right)\left[\left(X^{2}+Y^{2}\right)\right. \\
& \left.-\kappa_{3}\left(\delta-Z_{2}\right)\left(2 s_{3} \gamma_{3} K_{2}+Z_{2}\right)\left(2 s_{2} \gamma_{2} K_{2}-Z_{2}\right)\right] .
\end{aligned}
$$


Following Kummer [29, 21], the reduced equations may be written as $\dot{F}=\left\{F, H_{r}\right\}$ for the Poisson bracket

$$
\{F, G\}=\nabla \phi \cdot(\nabla F \times \nabla G) .
$$

The trajectories on the reduced surfaces are also obtained by slicing the surface with the planes $H_{r}=$ Constant. The Poisson structure on $\mathbb{C}^{3}$ drops to a Poisson structure on $\left(X, Y, Z_{1}, Z_{2}\right)$-space and this in turn induces the Poisson structure above. Correspondingly, the symplectic structure drops to one on each three-wave surface - this is an example of the general procedure of symplectic reduction (MMW reduction [30]). Notice however, that the three-wave surfaces may have singularities - this is because the group action is not free; this is one aspect of singular reduction. For the three-wave system a singular point appears on the three-wave surface when $\left|q_{1}\right|^{2} /\left(s_{1} \gamma_{1}\right)=\left|q_{3}\right|^{2} /\left(s_{3} \gamma_{3}\right)$ or equivalently $K_{1}=K_{2}$ and $K_{3}=0$. The geometry shows that that a homoclinic orbit passes through such a singular point-these are cut out by the plane $H_{r}=0$ when $H_{r}=-X$.

\section{Three-wave phase formulas}

The reduced dynamics determines the evolution of the wave intensities. Once it has been solved, the full dynamics of the three-wave system, including the wave phases, may be reconstructed.

In this section we consider the decay interaction, and, for definiteness, the particular case $\left(s_{1}, s_{2}, s_{3}\right)=(1,-1,1)$, epitomized by (1.4)-(1.6) (analysis of the other decay interactions is quite similar). In this case the reduced dynamics is typically periodic (the exceptions are fixed points, homoclinic orbits and heteroclinic orbits), but the full dynamics is not. Thus, after a period $T$ of the reduced dynamics, the wave intensities return to themselves while the phases are shifted.

The initial and final wave amplitudes are related by the phase symmetries (2.12)(2.13) (as remarked in Section 2, the third phase symmetry (2.14) is generated by the first two), so that

$$
\begin{aligned}
& q_{1}(T)=\exp \left(-i \Delta \phi_{1}\right) q_{1}(0), \\
& q_{2}(T)=\exp \left(-i \Delta \phi_{1}-i \Delta \phi_{2}\right) q_{2}(0), \\
& q_{3}(T)=\exp \left(-i \Delta \phi_{2}\right) q_{3}(0) .
\end{aligned}
$$

There are two methods for calculating the total phase shifts $\Delta \phi_{1}$ and $\Delta \phi_{2}$. The first, the traditional method, involves integrating the system by means of actionangle variables. One finds a canonical transformation from the wave amplitudes $q_{j}$ to new canonical coordinates, in which two of the generalized momenta are the constants of motion $K_{1}$ and $K_{2}$; their conjugate angles, $\phi_{1}$ and $\phi_{2}$, are then ignorable coordinates. Once the reduced dynamics is known, the total phase shifts $\Delta \phi_{1}$ and $\Delta \phi_{2}$ may be computed by integrating Hamilton's equations

$$
\dot{\phi}_{j}=\frac{\partial H}{\partial K_{j}},
$$


in which the Hamiltonian is expressed in terms of action-angle variables, over the reduced period $T$.

While straightforward in principle, in execution the traditional method is rather involved. In contrast, the alternate method of geometric phases (Marsden, Montgomery and Ratiu [31], Marsden [32], Shapere and Wilczek [33]), while requiring some additional theoretical machinery, leads in many cases to simpler calculations, as well as a suggestive geometric description of the phase shifts. Its application to the three-wave system, which may be viewed as a generalization of Montgomery's [14] analysis of rigid body rotation, is described in (Alber et al. [19]); here we give a brief account of the method and a summary of the results. For discussions of the well known geometric phases which appear in polarization optics (eg, Pancharatnam's phase), the reader is referred to (Shapere and Wilczek [33]) and (Bhandari [34]).

\subsection{The general case.}

The requisite additional theory is that of connections on principal bundles. For a discussion in the context of geometric phases and reduction, see (Marsden [32]) and the references therein. Here we shall proceed rather informally. A principal bundle $W$ is a manifold composed from two smaller ones, a base manifold $\bar{W}$ and a Lie group $G$. $W$ is constructed by attaching a copy of $G$ - a fiber - to each point of the base $\bar{W}$ in a particular way. $G$ acts on $W$ by group translation in the fibers. The simplest case, a trivial principal bundle, is where $W$ is just the Cartesian product $\bar{W} \times G$. A general principal bundle cannot be so expressed. While it is always possible to partition it into regions $U$ that are themselves cartesian products $\bar{U} \times G$ of neighborhoods $\bar{U}$ in the base with $G$, these regions may fit together in an interesting and nontrivial way, reflecting topological properties of the bundle.

Principal bundles typically arise in the following way. Suppose $G$ acts smoothly on a manifold $W$. Then $W$ is foliated into disjoint group orbits (ie, sets of points related to each other by group operations); the group orbits may be labelled by points in the quotient space $\bar{W}=W / G$. If $G$ acts freely (ie, $g \cdot w \neq w$ unless $g$ is the identity), then each group orbit is a copy of (ie, is diffeomorphic to) $G$. The quotient space $\bar{W}$ is a manifold, and $W$ may be regarded as a principal bundle with base $\bar{W}$, whose fibers are the group orbits.

This is precisely the situation which obtains in the theory of reduction of Hamiltonian systems with symmetry (Marsden and Weinstein [30]). For the three-wave system, the symmetry group is $T^{2}$, with action on phase space $\mathbb{C}^{3}$ given by (2.12)(2.13). Let $W \subset \mathbb{C}^{3}$ denote the level set $K_{1}=k_{1}, K_{2}=k_{2}$ of the momenta which generate the torus action. $W$ is invariant under the $T^{2}$-action, so that the Hamiltonian vector fields

$$
\begin{aligned}
& \xi_{1}=\left.\frac{d}{d \gamma}\right|_{0}\left(q_{1} \exp (-i \gamma), q_{2} \exp (-i \gamma), q_{3}\right), \\
& \xi_{2}=\left.\frac{d}{d \gamma}\right|_{0}\left(q_{1}, q_{2} \exp (-i \gamma), q_{3} \exp (-i \gamma)\right)
\end{aligned}
$$

generated by $K_{1}$ and $K_{2}$ are tangent to $W$. The $T^{2}$-action on $W$ is free provided 
$k_{1}$ and $k_{2}$ are not critical values of the momentum map. The critical values are $k_{1}=0, k_{2}=0$, and $k_{1}=k_{2}$, and their level sets require separate consideration (see Section 4.2 below).

Here we assume that $k_{1}$ and $k_{2}$ are positive and distinct, and, for definiteness, that $k_{1}>k_{2}$ (the alternative case $k_{2}>k_{1}$ is obtained just by exchanging indices). Then the torus action is indeed free, and $W$ is a principal $T^{2}$-bundle. The base manifold $\bar{W}$ is just the three-wave surface (3.5). We denote by $p: W \rightarrow \bar{W}$ the canonical projection.

More concretely, what this means is that we can introduce new coordinates $q_{j}=q_{j}\left(k_{1}, k_{2}, \phi_{1}, \phi_{2}, X, Y, Z_{2}\right)$ in terms of which the phase symmetries (2.12) and (2.13) are simply translations in the angles $\phi_{1}$ and $\phi_{2}$, respectively. $k_{1}$ and $k_{2}$ determine the values of the momenta, while $X, Y$ and $Z_{2}$ are coordinates on the three-wave surface (and therefore are constrained by (3.5)). However, any such system of coordinates necessarily has singularities. For fixed $k_{1}>k_{2}$, these are generically of Dirac-string type (ie, isolated points on the three-wave surface), and reflect the fact that $W$ is a nontrivial bundle.

A connection on $W$ is a pair of one-forms $A_{1}$ and $A_{2}$ satisfying the conditions

$$
\begin{aligned}
L_{\xi_{r}} A_{s} & =0, \\
\left.\xi_{r}\right\lrcorner A_{s} & =\delta_{r s},
\end{aligned}
$$

where $L_{X}$ and $\left.X\right\lrcorner$ respectively denote the Lie derivative and contraction with respect to $X$. A connection may be understood as a convention for resolving vectors in $W$ into components parallel and transverse to the fibers. Explicitly, the vertical (along the fiber) component $X_{v}$ of a vector $X$ is given by $\left.\left.(X\lrcorner A_{1}\right) \xi_{1}+(X\lrcorner A_{2}\right) \xi_{2}$; the horizontal (transverse to the fiber) component $X_{h}$ is given by $X-X_{v}$. The first condition (4.7) requires this decomposition to be invariant under the phase symmetries, while the second (4.8) requires that $X_{v}=X$ if $X$ is tangent to the fiber. These conditions do not determine the connection uniquely; the freedom lies in specifying which subspace of vectors is purely horizontal.

The exterior derivative of a connection, i.e. the pair of two-forms $V_{1}=d A_{1}$ and $V_{2}=d A_{2}$, is the curvature. Together with the homotopy formula $L_{X}=$ $X\lrcorner d+d X\lrcorner$, Eqs. (4.7) and (4.8) imply that $X\lrcorner V_{r}=0$ if $X$ is vertical; this in turn implies that the curvature projects down to a pair of two-forms $\bar{V}_{1}$ and $\bar{V}_{2}$ (also called the curvature) on the three-wave surface $\bar{W}$, uniquely defined by the condition

$$
p^{*} \bar{V}_{r}=V_{r}
$$

A fundamental result states that the integral of the curvature is an integer multiple of $2 \pi$, ie

$$
\oint_{\bar{W}} \bar{V}_{1}=2 \pi c_{1}, \quad \oint_{\bar{W}} \bar{V}_{2}=2 \pi c_{2} .
$$

The integers $c_{1}$ and $c_{2}$ are called the Chern numbers of the bundle. Nonzero 
Chern numbers imply the bundle is nontrivial. Explicit calculation gives

$$
\begin{aligned}
& c_{1}=0, c_{2}=-1, \quad \text { for } k_{1}>k_{2}>0, \\
& c_{1}=-1, c_{2}=0, \quad \text { for } k_{2}>k_{1}>0 .
\end{aligned}
$$

The decomposition of the total phase shifts (4.1)-(4.3) into geometric and dynamical contributions is defined in terms of the connection, as follows. Over a period $T$ of the reduced motion, a trajectory $q_{j}(t)$ of the three-wave equations describes a path $c^{\prime}$ in $W$ whose endpoints $q_{j}(0)$ and $q_{j}(T)$ lie in the same fiber. We connect the endpoints by a path $c^{\prime \prime}$ in the fiber (precisely how won't matter) to obtain a closed curve $c=c^{\prime}+c^{\prime \prime}$. See Fig 4.1.

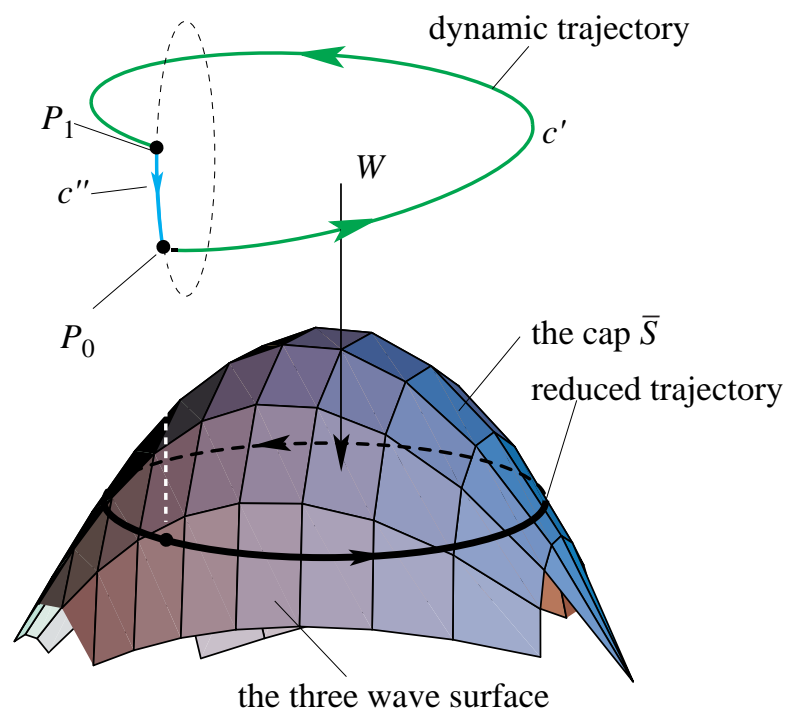

Figure 4.1: The geometry used to reconstruct solutions in $\mathbb{C}^{3}$ from periodic orbits on the three-wave surfaces is shown.

The integrals of the connection forms $A_{1}$ and $A_{2}$ along $c^{\prime \prime}$ yield, from (4.8), the phase shifts $-\Delta \phi_{1}$ and $-\Delta \phi_{2}$ (modulo $2 \pi$ ). Integrating the connection along the entire closed curve $c$, we obtain

$$
\begin{aligned}
\Delta \phi_{1} & =\Delta \phi_{1}^{d}+\Delta \phi_{1}^{g}, \\
\Delta \phi_{2} & =\Delta \phi_{2}^{d}+\Delta \phi_{2}^{g},
\end{aligned}
$$

where the dynamical phase $\Delta \phi_{r}^{d}$ is given by the integral of $A_{r}$ along the trajectory $c^{\prime}$, and the geometric phase $\Delta \phi_{r}^{g}$ by minus the integral of $A_{r}$ along the closed curve $c$.

Using Stokes' theorem, the geometric phases may be expressed as integrals $\int_{S} V_{r} d S$ of the curvature over a surface $S$ in $W$ bounded by $c$. With (4.9), this expression may be projected down to the three-wave surface to give $\Delta \phi_{r}^{g}=-\int_{\bar{S}} \bar{V}_{r} d \bar{S}$, where $\bar{S}$ is a surface bounded by the periodic orbit of the reduced dynamics. Expressed in this way, it is evident that the geometric phases depend only on the curve 
described by the reduced orbit and not on the rate at which this curve is traversed - in contrast, the dynamical phases $\left.\Delta \Phi_{r}^{d}=\int_{0}^{T} \dot{q}_{j}(t)\right\lrcorner A_{r} d t$ do depend on this rate. The geometric phases are the phase shifts (also called holonomy) accumulated along the horizontal lift $\tilde{q}_{j}(t)$ of the reduced orbit. (The horizontal lift $\tilde{q}_{j}(t)$ is a path in $W$ projecting onto the reduced orbit whose velocity $\dot{\tilde{q}}_{j}$ is horizontal, so that local rate of phase change, as defined by the connection, is zero). In other words, phase is parallel transported along $\tilde{q}_{j}(t)$. A nonvanishing dynamical phase reflects the fact that phase is not parallel transported along the dynamical trajectory $q_{j}(t)$.

It remains to specify a particular connection, which we call the kinematic connection. What distinguishes the kinematic connection is that it is defined purely in terms of the symplectic structure and the action of the symmetry group, and therefore is independent of the Hamiltonian. Thus it is characteristic of all systems defined on a given phase space $\left(\mathbb{C}^{3}\right.$ in the present case) invariant under a given symmetry (i.e. the torus action $(2.12)-(2.13))$. We shall give a general account in a subsequent paper, and confine our attention here to the three-wave system.

According to the symplectic reduction theorem (Marsden and Weinstein [30]), $\bar{W}$ is a phase space naturally endowed with a reduced symplectic form $\bar{\omega}$. The kinematic connection is defined (up to a choice of gauge) simply by requiring its curvature $\bar{V}_{r}$ to be proportional to $\bar{\omega}$. The constant of proportionality (which can depend on $k_{1}$ and $k_{2}$ ) is then determined by the Chern numbers (4.10), which gives

$$
\begin{aligned}
& \bar{V}_{1}=0, \\
& \bar{V}_{2}=-\frac{\bar{\omega}}{k_{2}} .
\end{aligned}
$$

for the kinematic curvature.

(The kinematic connection is related to the mechanical connection (Marsden [32]) for point symmetries on cotangent bundles; the mechanical curvature is also proportional to the reduced symplectic form. For point symmetries, however, the reduced phase space is typically unbounded, while the Chern numbers vanish.)

Using (4.15)-(4.16), the kinematic connection can be constructed explicitly; our calculations follow those in the Duistermaat-Heckman [35] theorems concerning the dependence of the reduced symplectic form on momentum. Omitting further details, we simply state the results.

The dynamical phases are given by

$$
\Delta \phi_{r}^{d}=\int_{0}^{T} \frac{\partial H}{\partial K_{r}} d t
$$

where the Hamiltonian $H$ is expressed in terms of coordinates $\alpha=\arg q_{1} \bar{q}_{2} q_{3}$ and $Z=-\left|q_{2}\right|^{2} /\left(2\left|\gamma_{2}\right| K_{2}\right)+\left|q_{3}\right|^{2} /\left(2 \gamma_{3} K_{2}\right)$ on the three-wave surface as well as the momenta $K_{1}$ and $K_{2}$, as follows:

$$
H\left(\alpha, Z, K_{1}, K_{2}\right)=-\left[\gamma_{1}\left|\gamma_{2}\right| \gamma_{3} K_{2}{ }^{3}(1-Z)(Z+1)\left(Z-\left(1-2 K_{1} / K_{2}\right)\right)\right]^{1 / 2} \cos \alpha .
$$

(It turns out to be more convenient to use $Z$, whose range $-1 \leq Z \leq 1$ is fixed, instead of $Z_{2}$.) If $Z$ and $\alpha$ were action-angle variables for the reduced dynamics, then 
(4.17) would represent the time integral of the frequencies in the angles conjugate to $K_{r}$. The geometric phases compensate for the fact that $Z$ and $\alpha$ are not action-angle variables. For them one obtains

$$
\begin{aligned}
\Delta \phi_{1}^{g} & =0, \\
\Delta \phi_{2}^{g} & =\frac{A(\bar{S})}{k_{2}} .
\end{aligned}
$$

Thus, the first geometric phase vanishes, while the second is proportional to the symplectic area $A(\bar{S})=\int_{\bar{S}} \bar{\omega}$ bounded by the reduced trajectory.

As is readily verified, the Hamiltonian (4.18) satisfies the relation $K_{1} \partial H / \partial K_{1}+$ $K_{2} \partial H / \partial K_{2}=3 H / 2$. With (4.17) this gives an identity for the weighted sum $K_{1} \Delta \phi_{1}^{d}+K_{2} \Delta \phi_{2}^{d}$ of the dynamical phases (a sum of actions),

$$
K_{1} \Delta \phi_{1}^{d}+K_{2} \Delta \phi_{2}^{d}=\frac{3}{2} H T .
$$

Together with (4.13)-(4.14) and (4.19)-(4.20), this yields the following phase identity for the total phase shifts:

$$
K_{1} \Delta \phi_{1}+K_{2} \Delta \phi_{2}=\frac{3}{2} H T+A(\bar{S}) .
$$

(The phase identity also follows directly from integration of the canonical one form defined below, as in [14].)

The foregoing analysis provides a natural and useful decomposition of the total phase shifts. The dynamical part can be computed without introducing actionangle variables, while the geometric part is simply an area enclosed on the threewave surface. The identity (4.22) is not easily obtained using traditional techniques. These are some of the advantages of the method of geometric phases.

\subsection{The case of second harmonic generation.}

The preceding analysis breaks down at critical values of the momentum. For example, the momentum level set $k_{1}=k_{2} \stackrel{\text { def }}{=} k$, where $\left|q_{1}\right|^{2} / \gamma_{1}=\left|q_{3}\right|^{2} / \gamma_{3}$, contains the fixed points $q_{1}=q_{3}=0$; at these fixed points, the torus action is not free (setting $\phi_{1}=-\phi_{2}$ in (2.12)-(2.13) leaves the fixed points invariant).

An important case is where $\gamma_{1}=\gamma_{3} \stackrel{\text { def }}{=} \gamma$. Then the three-wave equations (1.1)(1.3) (or, rather, the particular decay case we are considering) are invariant under the interchange symmetry $q_{1} \leftrightarrow q_{3}$, while the level set $k_{1}=k_{2}$ is itself invariant under interchange. On this level set, the wave amplitudes $q_{1}$ and $q_{3}$ differ by a phase factor $\exp i \beta$ which is constant in time. Letting $\left.q \stackrel{\text { def }}{=} \exp (-i \beta / 2) q_{1}=\exp (i \beta / 2) q_{3}\right)$, the three-wave equations reduce to

$$
\begin{gathered}
\frac{d q}{d t}=i \gamma \bar{q} q_{2}, \\
\frac{d q_{2}}{d t}=i\left|\gamma_{2}\right| q^{2} .
\end{gathered}
$$


This corresponds to the case of second harmonic generation (SHG) in nonlinear optics.

Like the three-wave equations, the SHG equations (4.23)-(4.24) may be written in Hamiltonian form. Taking the scaled Poisson bracket on $\mathbb{C}^{2}$ to be

$$
\{F, G\}=-2 i \gamma \frac{\partial F}{\partial q} \frac{\partial G}{\partial \bar{q}}-4 i\left|\gamma_{2}\right| \frac{\partial F}{\partial q_{2}} \frac{\partial G}{\partial \bar{q}_{2}}-(F \leftrightarrow G),
$$

the Hamiltonian is given by

$$
H_{\mathrm{SHG}}=-\frac{1}{4}\left(\bar{q}^{2} q_{2}+q^{2} \bar{q}_{2}\right)
$$

The SHG equations are invariant under the phase symmetry (i.e. circle-, or $S^{1}$ action)

$$
\left(q, q_{2}\right) \mapsto\left(q \exp (-i \phi), q_{2} \exp (-2 i \phi)\right),
$$

which is generated by the conserved momentum

$$
K=\frac{\left|q^{2}\right|}{2 \gamma}+\frac{\left|q_{2}^{2}\right|}{2\left|\gamma_{2}\right|} .
$$

(The bracket (4.25) is defined so that $K_{1}$ and $K_{2}$ coincide with $K$ on the degenerate level set.) Letting $W$ denote the level set $K=k$, we can apply reduction with respect to the circle action to obtain dynamics on the (two-dimensional) reduced phase space $\bar{W}=W / S^{1}$. The full dynamics may be reconstructed, and the total phase shift accumulated over a period decomposed into geometric and dynamical contributions, by following the general procedure described in Section (4.1). However, for the SHG system (and, more generally, for systems invariant under a circle action), a simpler calculation of the geometric and dynamical phases is obtained by integration of the canonical one-form [14], as we describe below. This case is also an example of discrete reduction as described in [32].

Let $c^{\prime}$ denote the curve described by the wave amplitudes $\left(q(t), q_{2}(t)\right)$ over a period $T$ of the reduced dynamics. The initial and final points are related by the total phase shift $\Delta \phi$, so that

$$
\left(q(T), q_{2}(T)\right)=\left(q(0) \exp (-i \Delta \phi), q_{2}(0) \exp (-2 i \Delta \phi)\right) .
$$

The initial and final conditions may be connected by a curve $c^{\prime \prime}$ generated by the circle action (4.27) so that $c=c^{\prime}+c^{\prime \prime}$ is a closed curve in in $\mathbb{C}^{2}$. Let $\Theta$ be a canonical one form on $\mathbb{C}^{2}$, i.e. a scaling of the Poincaré one form defined by

$$
\Theta=\frac{1}{4 i \gamma}(q d \bar{q}-\bar{q} d q)+\frac{1}{8 i\left|\gamma_{2}\right|}\left(q_{2} d \bar{q}_{2}-\bar{q}_{2} d q_{2}\right)
$$

with the property that $-d \Theta$ is the symplectic form $\omega$. By Stokes' theorem,

$$
\int_{c^{\prime}} \Theta+\int_{c^{\prime \prime}} \Theta=\int_{\Sigma} d \Theta
$$


where $\Sigma$ is a surface bounded by $c$ which projects down to a surface $\bar{\Sigma}$ bounded by the reduced orbit. (A more careful argument - as in holonomy theorems - shows that the existence of these surfaces is not necessary.)

Noting that $\int_{\Sigma} \omega=\int_{\bar{\Sigma}} \bar{\omega}$, where $\bar{\omega}$ is the reduced symplectic form, we obtain

$$
\int_{c^{\prime}} \Theta+\int_{c^{\prime \prime}} \Theta=-\int_{\bar{\Sigma}} \bar{\omega}
$$

Along the curve $c^{\prime \prime}$ generated by the symmetry, we get

$$
\int_{c^{\prime \prime}} \Theta=-k \Delta \phi
$$

while along the dynamical trajectory $c^{\prime}$ we get

$$
\left.\int_{c^{\prime}} \Theta=\int_{c^{\prime}}\left(\dot{q}, \dot{q}_{2}\right)\right\lrcorner \Theta d t=\frac{3}{2} H_{\mathrm{SHG}} T .
$$

Letting $A(\bar{\Sigma})$ denote the symplectic area of $\bar{\Sigma}$, we obtain from $(4.32)-(4.34)$ the expression

$$
\Delta \phi=\frac{3 H_{\mathrm{SHG}} T}{2 k}+\frac{A(\bar{\Sigma})}{k},
$$

for the total phase shift in the SHG dynamics. The first term is the dynamical phase $\Delta \phi^{d}$, and the second is the geometric phase $\Delta \phi^{g}$.

It is worth reconciling this result with the general results Comparison of (4.1)(4.3) and (4.29) gives the relation

$$
\Delta \phi=\frac{\Delta \phi_{1}+\Delta \phi_{2}}{2}
$$

between the total SHG and three-wave phase shifts. We obtain a similar relation for the geometric phases by setting $k_{1}=k$ and taking the limit $k_{2} \rightarrow k$ from below. This gives

$$
\lim _{k_{2} \rightarrow k^{-}} \frac{\Delta \phi_{1}^{g}+\Delta \phi_{2}^{g}}{2}=\frac{A(\bar{S})}{2 k}=\frac{A(\bar{\Sigma})}{k}=\Delta \phi^{g}
$$

(One has to check that $A(\bar{S})=2 A(\bar{\Sigma})$ in this limit.) Similarly, for the dynamical phases, the identity (4.21) gives

$$
\lim _{k_{2} \rightarrow k^{-}} \frac{\Delta \phi_{1}^{d}+\Delta \phi_{2}^{d}}{2}=\frac{3 H T}{4 k}=\frac{3 H_{\mathrm{SHG}} T}{2 k}=\Delta \phi^{d} .
$$

Thus, by passing from the three-wave equations to the SHG equations, we resolve the singularity on the three-wave surface which appears for $k_{1}=k_{2}$, and obtain a sensible continuation of the two geometric and dynamical phases $\Delta \phi_{r}^{g, d}$ across these critical values of the momentum. 


\section{The Lie-Poisson Structure}

In this section the three-wave equations are written on the dual of the Lie algebra of the group $\mathrm{SU}(3)$ or $\mathrm{SU}(2,1)$ using a Lie-Poisson structure. This Lie-Poisson system of equations has a quadratic rather than cubic Hamiltonian. The compatibility of the canonical and Lie-Poisson structures is discussed in the next section.

The Lie-Poisson description is obtained by recasting (1.1) as a differential equation in $\mathfrak{s u}(3)^{*}$, the dual of the Lie algebra of $\mathrm{SU}(3)$, for one of the decay interactions and a differential equation in $\mathfrak{s u}(2,1)^{*}$, the dual of the Lie algebra of $\mathrm{SU}(2,1)$, for the explosive interaction and the other two decay interactions.

Map to the dual of the Lie algebra. Define a map $U: \mathbb{C}^{3} \rightarrow \mathfrak{s u}(3)^{*}$ and a map $U: \mathbb{C}^{3} \rightarrow \mathfrak{s u}(2,1)^{*}$ as follows. Identify $\mathfrak{s u}(3)$ with $\mathfrak{s u}(3)^{*}$ using the standard Killing form:

$$
\langle A, B\rangle=\operatorname{Tr}(A B)
$$

Thus, $\mathfrak{s u}(3)^{*} \cong \mathfrak{s u}(3)$ is concretely realized as the space of complex skew Hermitian matrices with zero trace. The standard Killing form is also used to pair $\mathfrak{s u}(2,1)$ with $\mathfrak{s u}(2,1)^{*}$. While the resulting inner product remains nondegenerate in this case, it does become Lorenzian.

To obtain complex Hamiltonian systems for which the complex conjugate equations are self consistent we restrict the map so that

$$
U=-M U^{\dagger} M^{-1}
$$

where $M=\operatorname{diag}\left(m_{1}, m_{2}, m_{3}\right)$ and $m_{j}= \pm 1$. The map of $q=\left(q_{1}, q_{2}, q_{3}\right)$ to the matrix $U$ is then

$$
U=\left(\begin{array}{lll}
0 & q_{1} & q_{2} \\
-\frac{m_{2}}{m_{1}} \bar{q}_{1} & 0 & q_{3} \\
-\frac{m_{3}}{m_{1}} \bar{q}_{2} & -\frac{m_{3}}{m_{2}} \bar{q}_{3} & 0
\end{array}\right),
$$

where $U \in \mathfrak{s u}(3)^{*}$ for $\left(m_{1}, m_{2}, m_{3}\right)= \pm(1,1,1)$ and $U \in \mathfrak{s u}(2,1)^{*}$ otherwise.

Define a second map $Q_{1}: \mathbb{C}^{3} \rightarrow \mathfrak{s u}(3)$ or $Q_{1}: \mathbb{C}^{3} \rightarrow \mathfrak{s u}(2,1)$ as

$$
Q_{1}=\left(\begin{array}{lll}
0 & \alpha_{1} q_{1} & \alpha_{2} q_{2} \\
-\frac{m_{2}}{m_{1}} \alpha_{1} \bar{q}_{1} & 0 & \alpha_{3} q_{3} \\
-\frac{m_{3}}{m_{1}} \alpha_{2} \bar{q}_{2} & -\frac{m_{3}}{m_{2}} \alpha_{3} \bar{q}_{3} & 0
\end{array}\right)
$$

where $\alpha_{j} \in \mathbb{R}$. We take $\alpha_{1}>\alpha_{2}>\alpha_{3}>0$ throughout.

The three wave equations are written in matrix form as

$$
\frac{d U}{d t}=-\left[U, Q_{1}\right]
$$


where $[]:, \mathfrak{g} \times \mathfrak{g} \rightarrow \mathfrak{g}$ is the Lie bracket and in this context is equivalent to standard commutation of matrices. In component form these equations are:

$$
\begin{aligned}
\frac{d q_{1}}{d t} & =-\frac{m_{3}}{m_{2}}\left(\alpha_{2}-\alpha_{3}\right) q_{2} \bar{q}_{3}, \\
\frac{d q_{2}}{d t} & =-\left(\alpha_{3}-\alpha_{1}\right) q_{1} q_{3}, \\
\frac{d q_{3}}{d t} & =-\frac{m_{2}}{m_{1}}\left(\alpha_{1}-\alpha_{2}\right) \bar{q}_{1} q_{2} .
\end{aligned}
$$

Letting

$$
\begin{gathered}
\gamma_{1}=\left(\alpha_{2}-\alpha_{3}\right), \quad \gamma_{2}=\left(\alpha_{3}-\alpha_{1}\right) ; \\
\gamma_{3}=\left(\alpha_{1}-\alpha_{2}\right), \quad \text { and } \quad\left(s_{1}, s_{2}, s_{3}\right)=\left(m_{3} / m_{2},-1, m_{2} / m_{1}\right),
\end{gathered}
$$

one obtains the three-wave system (1.1) after $q_{k} \rightarrow i q_{k}$. Note that with this definition, $\sum \gamma_{k}=0$ automatically.

The quadratic invariants. The quadratic invariants (2.9)-(2.11) for (5.6)-(5.8) are

$$
\begin{aligned}
2 K_{1} & =\frac{m_{2}\left|q_{1}\right|^{2}}{m_{3}\left(\alpha_{2}-\alpha_{3}\right)}+\frac{\left|q_{2}\right|^{2}}{\left(\alpha_{1}-\alpha_{3}\right)}, \\
2 K_{2} & =\frac{\left|q_{2}\right|^{2}}{\left(\alpha_{1}-\alpha_{3}\right)}+\frac{m_{1}\left|q_{3}\right|^{2}}{m_{2}\left(\alpha_{1}-\alpha_{2}\right)}, \\
2 K_{3} & =\frac{m_{2}\left|q_{1}\right|^{2}}{m_{3}\left(\alpha_{2}-\alpha_{3}\right)}-\frac{m_{1}\left|q_{3}\right|^{2}}{m_{2}\left(\alpha_{1}-\alpha_{2}\right)} .
\end{aligned}
$$

If any two of these are positive or negative definite solutions are necessarily bounded. When this is not true solutions may blow up in finite time. If $m=\left(m_{1}, m_{2}, m_{3}\right)$ is $\pm(1,1,1), \pm(1,1,-1)$ or $\pm(1,-1,-1)$ the system corresponds to a decay interaction and if $m= \pm(1,-1,1)$ it is an explosive interaction. Notice that from the definition of the map $U$ one decay interaction is associated with $\mathfrak{s u}(3)$ and the other two as well as the explosive interaction are associated with $\mathfrak{s u}(2,1)$.

The quadratic Hamiltonian. The quadratic Lie-Poisson Hamiltonian is $H_{2}=$ $-\operatorname{Tr}\left(U Q_{1}\right) / 2$, and it has the explicit form

$$
H_{2}=\frac{m_{2}}{m_{1}} \alpha_{1}\left|q_{1}\right|^{2}+\frac{m_{3}}{m_{1}} \alpha_{2}\left|q_{2}\right|^{2}+\frac{m_{3}}{m_{2}} \alpha_{3}\left|q_{3}\right|^{2},
$$

This Hamiltonian can be written in terms of the quadratic invariants as

$$
H_{2}=2 \alpha_{1}\left(\alpha_{2}-\alpha_{3}\right) \frac{m 3}{m_{1}}\left(K_{1}+\beta K_{2}\right),
$$

where

$$
\beta=\left(\frac{\alpha_{3}}{\alpha_{1}}\right)\left(\frac{\alpha_{1}-\alpha_{2}}{\alpha_{2}-\alpha_{3}}\right)
$$


The Lie-Poisson bracket. As we show below, $Q_{1}=-\delta H_{2} / \delta U$, so we can write $d U / d t=\left[\delta H_{2} / \delta U, U\right]$. The general theory of Lie-Poisson structures is used to construct the Lie-Poisson bracket

$$
\{f, k\}_{1}(U)=-\left\langle U,\left[\frac{\delta f}{\delta U}, \frac{\delta k}{\delta U}\right]\right\rangle,
$$

where for $\mathfrak{g}=\mathfrak{s u}(3)$ or $\mathfrak{s u}(2,1), f, k: \mathfrak{g}^{*} \rightarrow \mathbb{R}, \delta f / \delta U, \delta k / \delta U \in \mathfrak{g}$, and $U \in \mathfrak{g}^{*}$.

Theorem 5.1 Choose $\gamma_{1}, \gamma_{3}>0$ and $\gamma_{2}<0$, or $\alpha_{1}>\alpha_{2}>\alpha_{3}>0$. With these definitions, the three-wave decay equations are Lie-Poisson equations on $\mathfrak{s u}(3)^{*}$ for $s=(1,-1,1)$ and $m= \pm(1,1,1)$; the three-wave decay equations are Lie-Poisson equations on $\mathfrak{s u}(2,1)^{*}$ for $s=(-1,-1,1)$ and $m= \pm(1,1,-1)$, or $s=(1,-1,-1)$

and $m= \pm(1,-1,-1)$; the explosive three-wave equations are Lie-Poisson equations on $\mathfrak{s u}(2,1)^{*}$ for $s=(-1,-1,-1)$ and $m= \pm(1,-1,1)$.

Proof Let $F: \mathfrak{g}^{*} \rightarrow \mathbb{R}$, then with the definitions above, $d F / d t=\left\{F, H_{2}\right\}$, or

$$
\left\langle\frac{\delta F}{\delta U}, \frac{d U}{d t}\right\rangle=-\left\langle U,\left[\frac{\delta F}{\delta U}, \frac{\delta H_{2}}{\delta U}\right]\right\rangle
$$

where $\langle$,$\rangle is defined by the trace as above. Now, D H_{2}(U) \cdot V=-\operatorname{Tr}\left(V Q_{1}(U)\right) / 2-$ $\operatorname{Tr}\left(U Q_{1}(V)\right) / 2$ for $V \in \mathfrak{g}^{*}$. We claim that $Q_{1}$ is a symmetric linear function of $U$. In fact, one can check directly that $Q_{1}(U)_{i, j}=c_{i, j} U_{i, j}$ (no sum), where $c_{i, j}$ is a symmetric matrix. Thus,

$$
\operatorname{Tr}\left(U Q_{1}(V)\right)=\operatorname{Tr}\left(\sum_{j} U_{k, j} c_{j, k} V_{j, k}\right)=\sum_{j, k} U_{k, j} c_{j, k} V_{j, k}=\operatorname{Tr}\left(Q_{1}(U) V\right) .
$$

Hence, $D H_{2}(U) \cdot V=-\operatorname{Tr}\left(V Q_{1}(U)\right)$ and so $\delta H_{2} / \delta U=-Q_{1}(U)$. Using this fact, write

$$
\left\langle\frac{\delta F}{\delta U}, \frac{d U}{d t}\right\rangle=-\left\langle\frac{\delta F}{\delta U},\left[U, Q_{1}\right]\right\rangle
$$

to obtain

$$
\frac{d U}{d t}=-\left[U, Q_{1}\right]
$$

It is checked that these indeed are the three-wave equations.

\section{Connections between the two Hamiltonian structures}

The three-wave equations have now been expressed using both the well known canonical Hamiltonian structure and the Lie-Poisson structure. In this section the relationship between them is discussed. A recursion relation is also produced and it is shown to be the same one obtained using the Lax approach. 
Second Hamiltonian structure. Modify the Lie-Poisson bracket for the threewave equations as follows:

$$
\{f, k\}_{0}(U)=-\left\langle U_{0},\left[\frac{\delta f}{\delta U}, \frac{\delta k}{\delta U}\right]\right\rangle
$$

in which the first matrix is fixed at $U_{0}$, where $U_{0} \in \mathfrak{s u}(3)^{*}$ or $U_{0} \in \mathfrak{s u}(2,1)^{*}$ is independent of $t$ and is to be specified. Taking $\delta f / \delta U$ and $\delta k / \delta U$ at $U$, this new bracket produces the equations of motion,

$$
\frac{d U}{d t}=\left[U_{0}, \frac{\delta k}{\delta U}\right]
$$

By choosing $U_{0}$ to be a constant diagonal matrix with $\operatorname{Tr}\left(U_{0}\right)=0$ and $k \propto H_{3}$, so that $\delta k / \delta U=Q_{2}, Q_{2}$ is quadratic in the $q_{i}$, we arrive at the three-wave equations. In this way the scaled canonical Hamiltonian structure is obtained directly from the Lie-Poisson bracket. Compatibility follows since this is a "translation of the argument" of the Lie-Poisson bracket, where $\{\}=,\{,\}_{1}(U)+\xi\{,\}_{0}\left(U_{0}\right)$ for an arbitrary real constant $\xi$. Both $\{,\}_{1}$ and $\{,\}_{0}$ are Poisson Brackets and the LiePoisson bracket with a shifted argument is also a Poisson bracket [36, 37]. The two three-wave brackets are therefore compatible.

Recursion relation. Having obtained the Lie-Poisson structure and the compatibility of the two Poisson brackets the recursion relation for the three-wave equations are found. Equate the two Poisson brackets and write

$$
\left\langle U_{0},\left[\frac{\delta f}{\delta U},\left(\frac{\delta k_{j+1}}{\delta U}\right)\right]\right\rangle=\left\langle U,\left[\frac{\delta f}{\delta U},\left(\frac{\delta k_{j}}{\delta U}\right)\right]\right\rangle .
$$

For this relation to hold the Lie brackets,

$$
\left[\left(\frac{\delta k_{j+1}}{\delta U}\right), U_{0}\right]=\left[\left(\frac{\delta k_{j}}{\delta U}\right), U\right]
$$

must also be equal. This is exactly the recursion relation obtained using the Lax approach. For the three-wave system it is invertible, and a complete set of $(\delta k / \delta U)_{j}$ is constructed.

The Lax equations. To demonstrate the connection with the Lax approach let $D, P, Q \in \mathfrak{s u}(3)$ or let $D, P, Q \in \mathfrak{s u}(2,1)$. Write

$$
\begin{aligned}
\lambda D & =[P, D], \\
\frac{d D}{d t} & =[Q, D] .
\end{aligned}
$$

Compatibility of these two equations leads to

$$
\frac{d P}{d t}+[P, Q]=0
$$


Let $P=\xi A+U$ and $Q^{(N)}=\sum_{j=0}^{N} Q_{j} \xi^{N-j}$, where $A, U, Q_{j} \in \mathfrak{s u}(3)$ or $A, U, Q_{j} \in$ $\mathfrak{s u}(2,1)$. Define $A$ to be $A=\operatorname{diag}\left(\beta_{1}, \beta_{2}, \beta_{3}\right)$ with $\sum_{k=1}^{3} \beta_{k}=0$. The $Q_{j}$ are general elements of the Lie algebra. As in (5.3), $U$ maps $\mathbb{C}^{3}$ into $\mathfrak{s u}(3)$ or $\mathfrak{s u}(2,1)$. With this definition for $P(6.7)$ becomes

$$
\frac{d U}{d t}+\xi\left[A, Q^{(N)}\right]+\left[U, Q^{(N)}\right]=0 .
$$

Now using the series for $Q^{(N)}$, the coefficients of powers of $\xi$ yield

$$
\begin{aligned}
\frac{d U}{d t}+\left[U, Q_{N}\right] & =0, \ldots \\
{\left[A, Q_{j}\right]+\left[U, Q_{j-1}\right] } & =0, \ldots \\
{\left[A, Q_{0}\right] } & =0 .
\end{aligned}
$$

The first equation is the integrable three-wave system. The second is the recursion relation. The final equation constrains the $Q_{j}$ so that $Q_{0} \in \operatorname{ker} \operatorname{ad}_{A}$. Letting $Q_{j}=(\delta k / \delta U)_{j}$ and $A=U_{0}$ this is exactly the recursion relation obtained using the method of Poisson pairs. The recursion relation implies that $\left[U, Q_{1}\right]=-\left[A, Q_{2}\right]$, so the three-wave equations are also written

$$
\frac{d U}{d t}=\left[A, Q_{2}\right]
$$

Carrying out the recursion (6.9)-(6.11) explicitly for the three-wave equations with $N=1$ and $\operatorname{diag}\left(Q_{j}\right)=0$ for $j>0$, it is found that with $Q_{0}=\operatorname{diag}\left(\beta_{1}^{0}, \beta_{2}^{0}, \beta_{3}^{0}\right)$,

$$
Q_{1}=\left(\begin{array}{lll}
0 & \frac{\beta_{2}^{0}-\beta_{1}^{0}}{\beta_{2}-\beta_{1}} q_{1} & \frac{\beta_{3}^{0}-\beta_{1}^{0}}{\beta_{3}-\beta_{1}} q_{2} \\
-\frac{m_{2}}{m_{1}} \frac{\beta_{2}^{0}-\beta_{1}^{0}}{\beta_{2}-\beta_{1}} \bar{q}_{1} & 0 & \frac{\beta_{3}^{0}-\beta_{2}^{0}}{\beta_{3}-\beta_{2}} q_{3} \\
-\frac{m_{3}}{m_{1}} \frac{\beta_{3}^{0}-\beta_{1}^{0}}{\beta_{3}-\beta_{1}} \bar{q}_{2} & -\frac{m_{3}}{m_{2}} \frac{\beta_{3}^{0}-\beta_{2}^{0}}{\beta_{3}-\beta_{2}} \bar{q}_{3} & 0
\end{array}\right),
$$

or

$$
\left(Q_{1}\right)_{i j}=\frac{\left(\beta_{i}^{0}-\beta_{j}^{0}\right)}{\left(\beta_{i}-\beta_{j}\right)} U_{i j} .
$$

Thus we find that $\alpha_{1}=\left(\beta_{2}^{0}-\beta_{1}^{0}\right) /\left(\beta_{2}-\beta_{1}\right), \alpha_{2}=\left(\beta_{3}^{0}-\beta_{1}^{0}\right) /\left(\beta_{3}-\beta_{1}\right), \alpha_{3}=$ $\left(\beta_{3}^{0}-\beta_{2}^{0}\right) /\left(\beta_{3}-\beta_{2}\right)$, so $Q_{1}$ is the map in (5.4). At the next iteration

$$
\left(Q_{2}\right)_{i k}=\sum_{j=1}^{3} \Gamma_{i j k} U_{i j} U_{j k}
$$

where

$$
\Gamma_{i j k}=\frac{1}{\beta_{i}-\beta_{k}}\left(\frac{\beta_{i}^{0}-\beta_{j}^{0}}{\beta_{i}-\beta_{j}}-\frac{\beta_{j}^{0}-\beta_{k}^{0}}{\beta_{j}-\beta_{k}}\right) .
$$


Note that $\Gamma_{i j k}$ is invariant under all permutations of its indices so we write $\Gamma=\Gamma_{i j k}$ and

$$
Q_{2}=\Gamma\left(\begin{array}{lll}
0 & -\frac{m_{3}}{m_{2}} q_{2} \bar{q}_{3} & q_{1} q_{3} \\
-\frac{m_{3}}{m_{1}} \bar{q}_{2} q_{3} & 0 & -\frac{m_{2}}{m_{1}} \bar{q}_{1} q_{2} \\
\frac{m_{3}}{m_{1}} \bar{q}_{1} \bar{q}_{3} & -\frac{m_{2}}{m_{1}} q_{1} \bar{q}_{2} & 0
\end{array}\right)
$$

Note that $\left[U, Q_{2}\right]=0$ terminating the recursion.

With these definitions, $d U / d t=\left[A, Q_{2}\right]$ yields

$$
\begin{aligned}
\frac{d q_{1}}{d t} & =-\frac{m_{3}}{m_{2}}\left(\beta_{1}-\beta_{2}\right) \Gamma q_{2} \bar{q}_{3} \\
\frac{d q_{2}}{d t} & =-\left(\beta_{3}-\beta_{1}\right) \Gamma q_{1} q_{3}, \\
\frac{d q_{3}}{d t} & =-\frac{m_{2}}{m_{1}}\left(\beta_{2}-\beta_{3}\right) \Gamma q_{2} \bar{q}_{1},
\end{aligned}
$$

which are the three-wave equations in (5.6)-(5.8) since $\left(\beta_{1}-\beta_{2}\right) \Gamma=\left(\alpha_{2}-\alpha_{3}\right)$, $\left(\beta_{3}-\beta_{1}\right) \Gamma=\left(\alpha_{3}-\alpha_{1}\right)$, and $\left(\beta_{2}-\beta_{3}\right) \Gamma=\left(\alpha_{1}-\alpha_{2}\right)$.

Conservation Laws and Hamiltonians. The $Q_{j}$ are gradients of Hamiltonian functions, and $Q_{j}=-\delta H_{j} / \delta U$, where the Hamiltonians

$$
H_{j+1}=-\operatorname{Tr}\left(U Q_{j}\right) /(j+1) .
$$

Here, $(j+1)$ is the highest power of $q_{k}$ in $H_{j+1}$. The cubic Hamiltonian defined here is proportional to the one associated with the scaled canonical structure from above. The quadratic Hamiltonian, $\mathrm{H}_{2}$, is associated with the Lie-Poisson structure.

These conserved quantities are found in a number of ways. The method of Poisson pairs produces invariants and their involutivity. The so called master conservation law is obtained by showing that the equation

$$
\left\langle U, \frac{d D}{d t}\right\rangle=\left\langle U,\left[Q^{(1)}, D\right]\right\rangle
$$

reduces to

$$
\frac{d}{d t}\langle D, U\rangle=\xi\left\langle D,\left[U, Q_{0}\right]\right\rangle
$$

Then using the recursion relation and in this case $D=Q^{(2)}$, one finds that $d\langle U, D\rangle / d t=$ 0 . In this way the Hamiltonians

$$
H_{2}=-\frac{1}{2}\left\langle Q_{1}, U\right\rangle, \quad H_{3}^{\prime}=-\frac{1}{3}\left\langle Q_{2}, U\right\rangle,
$$

are obtained, where $H_{3}^{\prime}=-2 i\left(m_{3} / m_{1}\right) \Gamma H_{3}$ if $q_{k} \rightarrow i q_{k}$. 


\section{Discussion}

Equations (6.5) and (6.6) provide alternate methods for solving the three-wave equations. They are used to construct the Lax pair of (6.7), which are linear equations for the evolution of an associated eigenfunction. Recall that as $D$ evolves, its determinant and the values of $\operatorname{Trace}\left(D^{k}\right)$ remain invariant. Since the coefficients of the spectral curve,

$$
\Gamma=\operatorname{Det}(D-y \mathbb{I})=0,
$$

involve only these quantities $\Gamma$ is also invariant. By constructing the Baker-Akheizer functions of the associated linear spectral problem or by constructing new coordinates using $D$, algebro-geometric methods are applied to integrate the system in terms of theta functions on Riemann surfaces.

Finally, recall that (6.7) is the Lax equation for $P$. If $P$ and $Q=Q^{(1)}$ are linear in $\xi$ then (6.7) contains the three-wave equations, as shown above; (6.7) is then the so called $\lambda$-representation for the three-wave equations (see $[20,38]$ ). The three-wave system exhibits a rich Hamiltonian structure that has only been partially discussed here. Note for instance that this system can be expressed in terms of the $R$-matrix

representation. Also note that the $\lambda$-representation for the three-wave equations is a reduction of the loop algebra associated with $\mathfrak{s u}(3)$ or $\mathfrak{s u}(2,1)$. A more complete treatment of the general structure of integrable equations of this type is found for instance in Refs. [36, 37, 39].

The family of $n$-wave interactions is connected to the groups $\mathrm{SU}(n)$ and $\mathrm{SU}(p, q)$. The structures described above for the three-wave example also follow for these higher-dimensional groups. Here integrability of the $n$-wave interaction on $\mathbb{C}^{n}$ is connected with the fact that there are a series of $\mathrm{U}(1)$ subgroups in $\mathrm{SU}(n)$ and $\mathrm{SU}(p, q)$ that reduce the equations on $\mathbb{C}^{n}$ to equations on surfaces in $\mathbb{R}^{3}$. In Kummer [21] the resonant Hamiltonian system with $n$-frequencies was analyzed using the reduction procedure discussed here for the three-wave system. Using $n-1$ independent $S^{1}$ symmetries the $n$-wave system is ultimately reduced to quadratures.

Solutions of the three-wave system analyzed here are also traveling wave or stationary solutions of an integrable partial differential equation (for solution of the partial differential equation see Refs. $[1,7,8,9,10,11,12,13])$. In this sense the integrable structure outlined above generalizes to the structure of the partial differential equation. More generally, each integrable system of ordinary differential equations is associated with a hierarchy of evolution equations through (6.5)-(6.6) by letting $\lambda \rightarrow \partial / \partial x, d / d t \rightarrow \partial / \partial t$ and associating $D, P$, and $Q$ with an appropriate group. For instance, the three-wave system is realized as an integrable PDE and the ODE system (1.1) gives traveling wave solutions. Further, the three-wave system is closely connected to the rigid body. The Euler equations are on the real subspace formed by taking $\mathfrak{s u}^{*}(3) \rightarrow \mathfrak{s o}^{*}(3)$. It follows that there is a related real partial differential equation for which the Euler equations are stationary or traveling wave solutions. 


\section{References}

[1] V.E. Zakharov and S.V. Manakov, Resonant interaction of wave packets in nonlinear media, Sov. Phys. JETP Lett. 18 (1973) 243-245.

[2] M.J. Ablowitz and H. Segur, Solitons and the Inverse Scattering Transform, (SIAM, Philadelphia, 1981).

[3] A.P. Fordy and D.D. Holm, "A tri-Hamiltonian formulation of the self-induced transparency equations," Phys. Let. A. 160 (1991) 143-148.

[4] J. Guckenheimer and A. Mahalov, Resonant triad interactions in symmetric systems, Physica D 54 (1992) 267-310.

[5] D. David and D.D. Holm, Multiple Lie-Poisson structures, reductions, and geometric phases for the Maxwell-Bloch traveling wave equations, J. Nonlinear Sci. 2 (1992) 241-262.

[6] G. B. Whitham, Linear and Nonlinear Waves (Wiley, New York, 1974).

[7] M.J. Ablowitz and R. Haberman, Resonantly coupled nonlinear evolution equations, J. Math Phys. 16 (1975) 2301-2305.

[8] D.J. Kaup, The three-wave interaction-a nondispersive phenomenon, Stud. Appl. Math. 55 (1976) 9-44.

[9] D.J. Kaup, A.H. Reiman and A. Bers, Space-time evolution of nonlinear threewave interactions. I. interaction in a homogeneous medium, Rev. Mod. Phys. 51 (1979) 275-309.

[10] D.J. Kaup, The solution off the general initial value problem for the full three dimensional three-wave resonant interaction, Proc. Joint US-USSR Symposium on Soliton Theory, Kiev, 1979, V. E. Zakharov and S.V. Manakov, eds., (NorthHolland, Amsterdam,1981) 374-395.

[11] V.E. Zakharov and S.V. Manakov, Soliton Theory, in Physics Reviews Soviet Scientific Reviews, ed. I. M. Khalatnikov, Section A 1 (1979) 133-190.

[12] A.C. Newell, Solitons in Mathematical Physics, (SIAM, Philadelphia, 1985).

[13] M.J. Ablowitz and P.A. Clarkson, Solitons, Nonlinear Evolution Equations and Inverse Scattering, (Cambridge University Press, Cambridge, 1991).

[14] R. Montgomery, How much does a rigid body rotate? A Berry's phase from the $18^{\text {th }}$ century, Am. J. Phys. 59 (1991) 394-398.

[15] C.J. McKinstrie, Relativistic solitary-wave solutions of the beat-wave equations, Phys. Fluids 31 (1988) 288-297.

[16] C.J. McKinstrie and G.G Luther, Solitary-wave solutions of the generalised three-wave and four-wave equations, Phys. Lett. A 127 (1988) 14-18. 
[17] C.J. McKinstrie and X.D. Cao, The nonlinear detuning of three-wave interactions, J. Opt. Soc. Am. B 10 (1993) 898-912.

[18] M.S. Alber, G.G. Luther and J.E. Marsden, Complex billiard Hamiltonian systems and nonlinear waves, in: A.S. Fokas and I.M. Gelfand, eds., Algebraic Aspects of Integrable Systems: In Memory of Irene Dorfman, Vol. 26 of Progress in Nonlinear Differential equations (Birkhäuser, 1997) 1-15.

[19] M.S. Alber, G.G. Luther, J.E. Marsden, and J.M. Robbins Geometric phases in the three-wave equations, (1998) in preparation.

[20] S.V. Manakov, Note on the integration of Euler's equations of the dynamics of and n-dimensional rigid body, Func. Anal and its Apps 10 (1976) 328-329.

[21] M. Kummer, On resonant classical Hamiltonians with $n$ frequencies, J. Diff. Eqns. 83 (1990) 220-243.

[22] D. David, D.D. Holm and M.V. Tratnik, Integrable and chaotic polarization dynamics in nonlinear optical beams, Phys. Lett. A 137 (1989) 355-364.

[23] E. Knobloch, A. Mahalov and J.E. Marsden, Normal forms for threedimensional parametric instabilities in ideal hydrodynamics, Physica D $\mathbf{7 3}$ (1994) 49-81.

[24] V. Kirk, J.E. Marsden and M. Silber, Branches of stable three-tori using Hamiltonian methods in Hopf bifurcation on a rhombic lattice, Dyn. and Stab. of Systems, 11 (1996) 267-302.

[25] G. Haller and W. Wiggins, Geometry and chaos near resonant equilibria of 3-DOF Hamiltonian systems, Physica D 90 (1996) 319-365.

[26] M.S. Alber, G.G. Luther, J.E. Marsden and J.M. Robbins, Geometry and control of $\chi^{(2)}$ processes and the generalized Poincaré sphere, submitted to Opt. Lett.

[27] R. Cushman and D.L. Rod, Reduction of the semisimple 1:1 resonance, Physica 6D (1982) 105-112.

[28] M. Born and E. Wolf, Principles of Optics, (Pergamon, Oxford, 1980).

[29] M. Kummer, An interaction of three resonant modes in a nonlinear lattice, J. Math. Anal. and Apps. 52 (1975) 64.

[30] J.E. Marsden and A. Weinstein, Reduction of symplectic manifolds with symmetry, Rep. Math. Phys. 5 (1974) 121-130.

[31] J.E. Marsden, R. Montgomery and T.S. Ratiu, Reduction, symmetry, and phases in mechanics, Memoirs AMS 436 (1990).

[32] J.E. Marsden, Lectures on Mechanics, (Cambridge: Cambridge University Press, 1992). 
[33] A. Shapere and F. Wilczek, eds. Geometric Phases in Physics, (Singapore: World Scientific, 1989).

[34] R Bhandari Polarization of light and topological phases, Phys. Rep. 281 (1997) $2-64$.

[35] J.J. Duistermaat, and G.J. Heckman On the variation in the cohomology of the symplectic form of the reduced phase space, Invent. math. 69 (1982) 259-268.

[36] V.I. Arnold and A.B. Givantal, Symplectic geometry Itogi Nauki. Fundamentalnye Napravleniya 4 (1985) VINITI, Moscow, 5-139; English transl.: Encyclopedia of Math. Sci. 4 (1990) Springer-Verlag 1-136.

[37] V.V. Trofimov and A.T. Fomenko, Geometric and algebraic mechanisms of the integrability of Hamiltonian systems on homogeneous spaces and Lie algebras, Itogi Nauki. Fundamentalnye Napravleniya 16 (1987), VINITI, Moscow; English transl: Encyclopedia of Math. Sci. 16 (1994), Springer-Verlag, 261-333.

[38] S.P. Novikov, Solitons and Geometry, (Academia Nazionale dei Lincei and the Scuola Normale Superiore, Press Syndicate of the University of Cambridge, Cambridge, 1994).

[39] Dynamical systems VII, eds V.I. Arnold and S.P. Novikov, Itogi Nauki. Fundamentalnye Napravleniya 16 (1987), VINITI, Moscow; English transl: Encyclopedia of Math. Sci. 16 (1994), Springer-Verlag. 